\title{
Chest nodule in a patient with multiple myeloma
}

\author{
Bina E. Franklin • Mihaela Oprea • \\ Sona M. Franklin
}

Received: 24 September 2009/Revised: 11 January 2010/ Accepted: 24 January 2010/Published online: 16 February 2010

(C) The Japanese Society of Hematology 2010

A 79-year-old African American male with multiple myeloma with bony metastasis was admitted to our hospital for failure to thrive. The patient was diagnosed with multiple myeloma 2 years earlier. Physical exam revealed a $2 \times 2$ in. nodular mass on the right anterior chest wall $5 \mathrm{~cm}$ below the clavicle. The mass commenced as three small dots, which became confluent and grew over the past 2 months to attain present size (see Fig. 1). The mass was found to be immobile, fungating, lobulated, and hard. The skin on its surface could not be pinched.

A biopsy of the lesion was conducted which revealed CD138 (plasma cells and epithelial cell subset), kappa (immunoglobulin light chain kappa), IgA and IgG (immunoblobulin heavy chain). Bone marrow biopsy was positive for the following: kappa, IgA (plasma cell subset) and CD138. Analysis revealed $0.4 \%$ of abnormal cells in the bone marrow, interpreted as a plasma cell dyscrasia. Myeloma cells were negative for $13 q 14$ deletion and $\mathrm{t}(11: 14)$ translocation. Further laboratory evaluation revealed the patient's hemoglobin to be $6.9 \mathrm{~g} / \mathrm{dL}$ and hematocrit was $20.1 \%$. Previous evaluation of calcium levels were within normal limits.

The patient had been undergoing treatment with thalidomide for multiple myeloma. But development of

B. E. Franklin · M. Oprea

Department of Internal Medicine,

The Mount Vernon Hospital, 12 North 7th Street,

Mount Vernon, NY 10550, USA

B. E. Franklin $(\bowtie)$

P.O. Box 7169, Elkins Park, PA 19027, USA

e-mail: binafranklin@hotmail.com

S. M. Franklin

College of Medicine, American University of Antigua,

2 Wall Street, 10th Floor, New York, NY 10005, USA bradycardia and hypotension led to cessation of thalidomide treatment. Development of the nodular mass then ensued and the patient expired shortly thereafter.

Given the patient's bone marrow status, and the common antibodies on immunohistochemistry between the marrow and the chest lesion, it is likely that the lesion developed as an extension from the bone.

Though our patient developed the lesion shortly after cessation of thalidomide, it may be possible that thalidomide itself contributed to its development initially. Thalidomide has been reported to target marrow stromal cells, alter cytokine production of interleukin- 6 and tumor necrosis factor alpha, and reduce the adhesion of malignant plasma cells. Malignant plasma cells may become resistant to therapy and may disseminate and infiltrate peripheral tissues as a result of modified cell-to-cell contact and interactions in the bone marrow microenvironment [1].

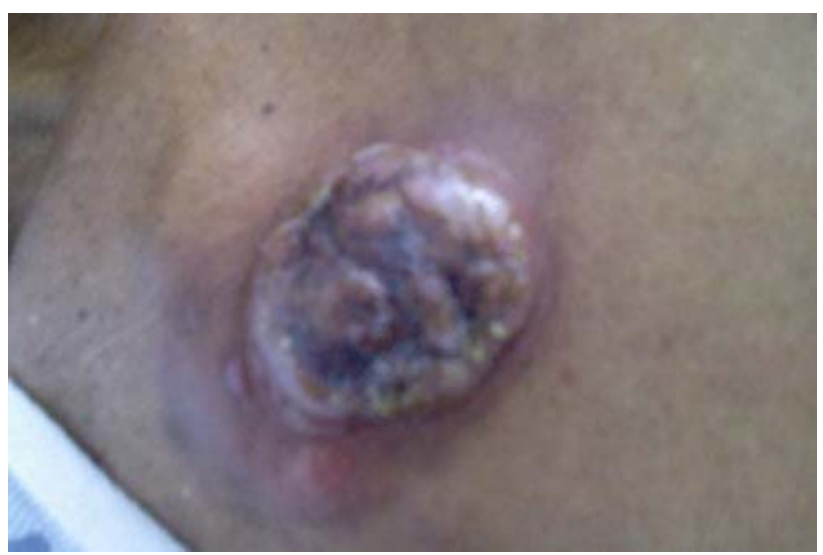

Fig. 1 Nodule that developed on the patient's chest wall after stopping treatment with thalidomide 
We report a very rare cutaneous manifestation of multiple myeloma. These lesions generally portend a poor prognosis, as was the case with our patient.

Conflict of interest statement The authors have no conflicts of interest to disclose.

\section{Reference}

1. Alexandrescu DT, Koulova L, Wiernik PH. Unusual cutaneous involvement during plasma cell leukaemia phase in a multiple myeloma patient after treatment with thalidomide: a case report and review of the literature. Clin Exp Dermatol. 2005;30(4):391-4. 